\title{
STUDY OF Si(111) IMPLANTED WITH As IONS BY X-RAY DIFFRACTION AND GRAZING INCIDENCE METHODS
}

\author{
J.B. Pelka, J. Górecka, J. Auleytner, J. Domagala \\ AND J. BĄK-MISIUK \\ Institute of Physics, Polish Academy of Sciences \\ Al. Lotników 32/46, 02-668 Warsaw, Poland
}

The Si(111) wafer cut from a bulk single crystal obtained by the Czochralski method was implanted with $5 \times 10^{16} \mathrm{I} \mathrm{cm}^{-2}$ of As ions of energy $80 \mathrm{keV}$. The dose applied was chosen above the amorphization limit of the silicon substrate. Two samples, implanted and a reference, were studied by grazing incidence X-ray reflectometry and X-ray diffraction methods using a high resolution Philips MRD system equipped with a $\mathrm{Cu}$ source and a channel-cut monochromator. The obtained spectra were compared with distributions of ion range and defect production calculated with TRIM program [1], as well as with theoretical models of reflectivity $[2,3]$. The results of grazing incidence $\mathrm{X}$-ray reflectometry reflectivity of the implanted sample show well-pronounced oscillations, which can be associated with a layer about $50 \mathrm{~nm}$ thick, approximately comparable to the thickness of the defected layer estimated from the TRIM method. Theoretical calculations of reflectivity clearly indicate an occurrence of a $\mathrm{Si}$ layer of electron density lower about $10-15 \%$ comparing to the unimplanted Si sample. This can be due to the vacancy production during ion implantation. A comparison of the spectra with a density distribution profile concluded from the TRIM calculations shows large discrepancies. The results indicate the applicability of grazing incidence X-ray reflectometry method in a study of amorphization processes in implanted layers.

PACS numbers: 68.55.Jk, 68.55.Ln

\section{Introduction}

Various aspects of the implantation process in silicon wafers have extensively been studied for long by a range of methods. However, most of these methods, especially those based on X-ray diffraction, are suitable to study the implanted materials in their crystalline state. Therefore, knowledge of material structure and implantation processes in amorphous layers, is not always complete, despite its primary importance in material science and technological processes. 
The grazing incidence X-ray reflectometry (GIXR) has for long been successfully applied to the study of various layered structures; especially in the last decade, due to a substantial effort in construction of X-ray devices, it is getting to be a standard tool in laboratories dealing with thin film structures. The X-ray reflectivity at grazing incidence for a multilayer system was first described by Parrat [2]. He formulated a recursive algorithm based on the Fresnel equation which enabled one to calculate the theoretical reflectivity of a multilayer structure, and showed that it is possible to calculate also the reflectivity from a layer of arbitrary electron density profile by "slicing" it onto a set of sublayers with fixed electron densities. A similar algorithm was used in this work to fit the experimental data [3]. Any differences in GIXR reflectivity are produced by changes in electron density of the material independently of its crystalline state. Therefore it can serve as an excellent, non-destructive tool to study in-depth electron density profiles in crystalline or amorphous layers with thicknesses $1 \mathrm{~nm}$ - few hundreds $\mathrm{nm}$. In addition, it can provide information about surface and interfacial roughness [5, 6].

An application of the technique to the study of implantation processes is, despite the above, not very common nowadays. The usefulness of the method was shown, e.g., by Bahr et al. [4], who used GIXR and diffuse scattering to study the implantation processes in $\mathrm{CoSi}_{2}$ layer in $\mathrm{Si}$ produced by the ion-beam synthesis. They presented a quantitative evaluation of the diffuse scattering underneath the specular reflectivity for this system and found strongly varying electron densities. However their study was for crystalline samples, as their implantation was with substrates at elevated temperatures to prevent amorphization.

A study of $\mathrm{Si}(111)$ implanted with the dose of As ions exceeding the limit of amorphization, which is the main aim of this work, gives also opportunity to discuss the usefulness of the GIXR reflectivity technique when applied to the thin amorphous layers obtained during the implantation processes.

\section{Experimental}

Studies were carried out on a perfect silicon wafer of (111) orientation implanted by As ions at an energy of $80 \mathrm{keV}$ and with a dose $5 \times 10^{16} \mathrm{I} \mathrm{cm}^{-2}$. The implantation process was kept at room temperature. Before implantation the wafer was examined by the $\mathrm{X}$-ray topography method which did not reveal any extended defects. A part of the wafer was left unimplanted serving as a reference sample. The defect structure of the implanted wafer was studied using high resolution diffractometry in a triple crystal configuration. The main factors influencing the crystal perfection are mosaicity and fluctuation of the lattice parameter. The reciprocal space map allows one to separate the both effects. Therefore reciprocal space mapping was performed for the implanted and unimplanted part of the wafer.

The reflectivity measurements were recorded at the Institute of Physics, Polish Academy of Sciences, Warsaw, with the $\mathrm{Cu} K_{\alpha}$ radiation by a high performance MRD Philips diffractometer equipped with a $\mathrm{Si}$ (440) channel-cut monochromator and a $\mathrm{Ge}(1,-1)$ analyzer. The horizontal divergence of the device was about 12 arc seconds $\left(0.0033^{\circ}\right)$. In order to limit the beam spot on the sample at lower angles of incidence an additional slit $50 \mu \mathrm{m}$ wide was applied between the monochromator 


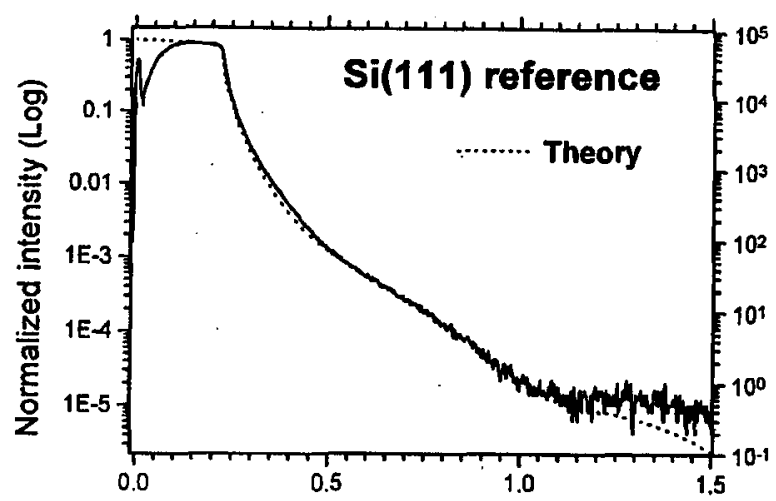

Fig.1

angular position [degrees]

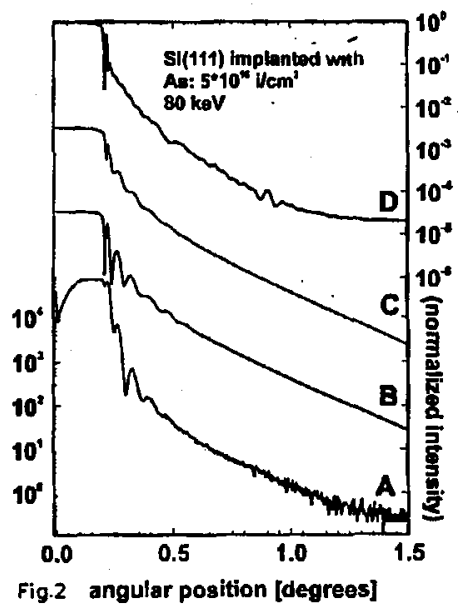

Fig. 1. Reflectivity of reference sample. Solid line - experimental data, dotted line theoretical fit.

Fig. 2. Reflectivity of implanted sample. A - experimental data, B,C,D - theoretical calculations with various density profile models defined in Fig. 3.

and the sample. The spectra were recorded in the angular range of $0-2.2^{\circ}$. The obtained reflectivity for a reference $\mathrm{Si}(111)$ sample is shown in Fig. 1 (solid line) and for an implanted sample in Fig. 2 (curve A). The intensity scales in both figures are logarithmic. The signal/noise ratio in these measurements was higher than $10^{5}$.

\section{Results and discussion}

As it was mentioned above, the wafer was examined before the implantation by the X-ray topography which did not reveal any extended defects. From Fig. 1 we can see that the experimental reflectivity differs from that expected for a pure silicon. A trace of oscillation with a long period and a small amplitude suggests that on its surface there exists an additional ultra thin layer. The theoretical fit assuming the $6.3 \mathrm{~nm}$ layer of $\mathrm{SiO}_{x}$ is compared to the experimental reflectivity in Fig. 1 (dotted line). It was obtained assuming the oxide layer to be composed. of two sublayers: the "surface" sublayer $3 \mathrm{~nm}$ thick with a lower mass density $\left(2 \mathrm{~g} / \mathrm{cm}^{3}\right)$ and the deeper layer of a slightly higher density $\left(2.15 \mathrm{~g} / \mathrm{cm}^{3}\right)$. The overall similarity of the experimental and the theoretical curves is very good; the discrepancy above the $1.1^{\circ}$ of angle of incidence is due to the fact that in the theoretical curve the signal/noise ratio of the device has not been taken into account. The roughness of the top surface was estimated to be about $0.8-0.9 \mathrm{~nm}$ rms. It has to be stressed that the oxide layer on the wafer during implantation was thinner, probably under $3 \mathrm{~nm}$; it is indicated by an earlier measurement (not shown here), which was performed with a lower accuracy, and up to the angle of incidence $0.8^{\circ}$ only. The increase of the oxide layer on the wafer we ascribe to a further treatment, like cleaning after some measurements in organic solvents. 
The 115 reciprocal space map for the implanted part of the wafer has shown, compared to the unimplanted one, the existence of lattice parameter fluctuation in the direction of a higher lattice parameter. These changes are probably caused by strain between the bulk crystal and the implanted layer. The reflectivity of the implanted layer (see Fig. 2, curve A) shows well-pronounced oscillations; from the positions and amplitudes of the observed 6 maxima we can derive that the total thickness of the layer (or layers) which can produce them is about $50 \mathrm{~nm}$; and the material of the layers is of a lower electron density comparing to the pure silicon. The latter conclusion is also supported by an observed small shift in the critical angle comparing to the situation in the pure Si. In order to extract more information from the measured reflectivity, theoretical calculations for a variety of density profiles were performed and compared with the experimental data.

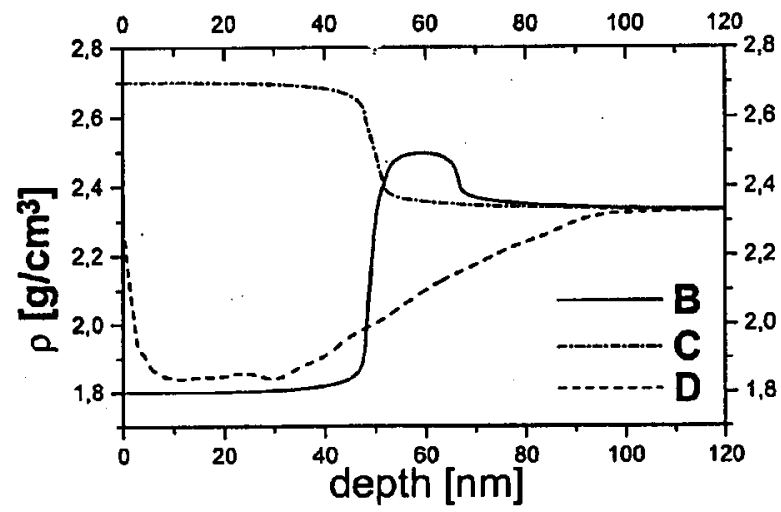

Fig. 3. Density distribution profiles applied to the reflectivity calculations in Fig. 2. B - bilayer model, first layer of lower density; $\mathrm{C}$ - single layer of higher density, D model extracted from the TRIM calculations.

The starting point for these models was calculation of an ion range and vacancy distribution, which has been done using the Monte-Carlo algorithm known as TRIM [1]. Both of the distributions are in qualitative agreement with the concluded layer thickness. We assumed that the lowering of the electron density in the density profile is due to the massive vacancy production during the implantation process. From the vacancy distribution profile obtained by TRIM we constructed the density profile which is shown in Fig. 3 (curve D). Then, after slicing it onto 24 sublayers, the theoretical reflectivity was calculated, and the result is shown in Fig. 2 (curve D). Comparing this result to the experimental reflectivity (curve A in Fig. 2), we can see that it gives a smaller number of oscillations with a much lower period, and additional small peaks at higher angles occur.

The curve $\mathrm{C}$ in Fig. 2 is obtained assuming that the $50 \mathrm{~nm}$ layer is composed of pure As of the mass density about $2.4 \mathrm{~g} / \mathrm{cm}^{3}$. The appropriate density profile used for calculations is shown in Fig. $3 \mathrm{C}$. This was to testify, whether a layer of a higher density would not explain the observed reflectivity; the applied ion dose of $5 \times 10^{16} \mathrm{I} \mathrm{cm}^{-2}$ implies that if all ions are collected uniformly in the layer $50 \mathrm{~nm}$ 
thick, the ratio of Si:As atoms would be approximately about 5:1 in such a layer. As this model did not fit to the experimental result due to small amplitudes of calculated oscillations (it is not shown here), we increased still the density of As atoms in a layer. However even in this case we can see that the fit is far from the experimental result, both in oscillation amplitudes, and in the critical angle, which is higher an the fitted curve. In general, all models assuming the density close to the surface equal to the pure $\mathrm{Si}$ or higher did not fit well to the experimental reflectivity.

The curve B in Fig. 2 was obtained with a profile shown in Fig. 3 (curve B). It was composed of two sublayers, the first one, just under the surface, was assumed to be $\mathrm{Si}$ of a lower density and a thickness of $50 \mathrm{~nm}$; the next layer was silicon of a higher density and a thickness of $14 \mathrm{~nm}$. The two interfaces: the one between the two layers, and the other between the higher density layer and the unaffected substrate layer were assumed to be of a smoothly varying density in the width of the order 5-6 nm. Comparing the experimental data we see that this model explains well not only positions of all peaks, but also amplitudes of four (out of six) last peaks, and the shape of the reflectivity at higher angles. When convoluted with the apparatus function of 12 arc seconds FWHM also the first minimum depth is better fitted. However, the relative intensities in the first two peaks are not well explained by this model. It suggests that the model is oversimplified, and the true electron density profile is more complicated.

We calculated also a number of theoretical reflectivities (not shown here) assuming other, more sophisticated models of density profile. In some cases we obtained good fits to the first two or three peaks, loosing however the fit at higher angles. All this work indicates clearly that to explain the obtained reflectivity we have to assume that just under the surface there is a layer of a substantially lower density comparing to the pure Si wafer (about 10-15\%); in the deeper parts of the layer there exists another layer with a density higher than the pure Si. Between these two layers there exists an interfacial layer with a thickness of the order 5-6 nm, where the density profile smoothly varies. However, the exact density profile of the layers can be pretty complicated and we were not able to estimate it correctly in this work.

\section{Conclusions}

In the present paper the GIXR reflectivity measurements were applied to study the Si layers affected by the implantation with the As ions of the energy $80 \mathrm{keV}$ and the dose of $5 \times 10^{16} \mathrm{I} \mathrm{cm}^{-2}$, which was chosen above the amorphization limit. The results were compared with a number of theoretical calculations assuming various models of in-depth density distribution in the affected layers. It has been found that the model derived from the Monte-Carlo TRIM algorithm agrees with the experiment only in an approximate way, providing a similar thickness of layers disturbed by implantation. The density distribution from the TRIM model gives a too shallow slope of the deeper tail, comparing to the situation concluded from the experimental reflectivity. The most probable reason for this is that this deeper tail of the distribution is increasing rapidly (within about 4-6 $\mathrm{nm}$ interface layer) and forms a sublayers with a higher electron density comparing to the 
pure Si. This higher density layer can be constituted both of As atoms and $\mathrm{Si}$ atoms in interstitial positions.

Although we were not able to obtain the exact density profile directly from the recorded reflectivity, it was however possible to verify various models of this profile, and to obtain many partial indications concerning the structure of the implanted layers. This shows an extreme usefulness of this method in studying the layers affected by implantation process, crystalline or amorphous.

\section{Acknowledgments}

This work was supported in part by the Committee for Scientific Research, grant No. 2P03B 02109 and No. 2P302 083 05. The Phillips MRD diffractometer used in this work was founded by the Foundation for Polish Science (within the program SEZAM 94).

\section{References}

[1] J.F. Ziegler, J.P. Biersack, U. Littmark, The Stopping and Range of Ions in Solids, Pergamon Press 1985.

[2] L.G. Parratt, Phys. Rev. 95, 359 (1954).

[3] J.B. Pełka, S. Lagomarsino, W. Jark, J. Domagała, Acta Phys. Pol. A 89, 323 (1996).

[4] D. Bahr, W. Press, R. Jebasinski, S. Mantl, Phys. Rev. B 47, 4385 (1993).

[5] S.K. Sinha, Acta Phys. Pol. A 89, 219 (1996).

[6] D.K. Bowen, M. Wormington, Adv. X-ray Anal. 38, 171 (1993). 